\title{
EL ANÁLISIS DE EQUIVALENCIA VALOR-VALOR EN LA EVALUACIÓN DE DAÑOS AMBIENTALES. UNA APLICACIÓN A FUEGOS FORESTALES EN ESPAÑA
}

\author{
Ph.D. Riera, Pere ${ }^{1}$
}

Dr. Borrego, Armonía ${ }^{2}$

\begin{abstract}
Resumen
La legislación de varios países establece que cuando cierta actividad económica causa un determinado daño ambiental, el responsable debe reparar el medio hasta llevarlo de nuevo a su estado básico. Sin embargo, mientras este no se restablece completamente, los recursos o servicios que dejan de proveerse (daño temporal) deben también compensarse. El cálculo de esta compensación se realiza mediante los Ilamados análisis de equivalencia. En su versión valor-valor, el responsable debe realizar una mejora ambiental de valor social equivalente al del daño temporal que sufre la sociedad. Este artículo muestra un ejemplo de análisis de equivalencia valor-valor para un gran incendio forestal en España, con dos aplicaciones del método de valoración contingente. El resultado muestra que por cada hectárea de bosque quemado, el responsable debe restablecer dicha hectárea de bosque y proveer aproximadamente una tercera parte más para compensar en equivalencia el daño temporal.
\end{abstract}

Palabras claves: Valoración contingente, análisis de equivalencia, incendios forestales, responsabilidad ambiental.
Abstract

Several countries have established guidelines for the assessment of damages to natural resources requiring the party causing the damage to repair the environment, returning it to the baseline. Furthermore, the interim losses in resources or services until the baseline is regained also have to be compensated for. The estimation of the amount to be compensated is the object of the equivalency analyses. The value-to-value approach of the equivalency analysis requires that the value to society of the compensation improvement has to offset the social value of the interim loss. This article provides a value-to-value example for a forest fire in Spain, with two contingent valuation applications. Results show that for every hectare of forest burned, circa a third of a hectare has to be provided above the baseline to compensate for the interim losses.

JEL classification: Q23 forestry; Q51 valuation of environmental effects; C93 field experiments.

Keywords: Contingent valuation, equivalency analysis, forest fires, environmental responsibility. 


\section{Introducción}

Ante determinados daños ambientales, la legislación aplicable en Estados Unidos (CERCLA, 1980, OPA, 1990), países de la Unión Europea (Council of the European Parliament, 2004) y otros, prevé que se realicen análisis de equivalencia. El causante (u operador, en el argot de esta literatura) del daño ambiental (incidente) debe reparar completamente el medio (reparación primaria) hasta dejarlo en el nivel de calidad que tendría de no haberse dado el incidente (estado básico). En caso de que no fuera viable dicha recuperación, el operador debe realizar una reparación complementaria de forma que al final no haya diferencia sensible entre el estado real del medio ambiente y su estado básico. Sin embargo, no es suficiente con volver al estado básico. Mientras eso no llega, se sufren unos daños temporales. A lo mejor el bosque tarda unas décadas en volver al estado de madurez correspondiente, o la población de peces del lago unos meses en recuperarse. Estos daños temporales, también llamados débitos, deben a su vez compensarse con una mejora ambiental (créditos) equivalente a cargo del operador; es la llamada reparación compensatoria. E cálculo de los débitos y los créditos equivalentes recibe el nombre de análisis de equivalencia. En definitiva la idea es que la sociedad no note al final ninguna pérdida, ni ganancia, en calidad ambiental respecto a la ausencia del incidente. O en términos de la legislación norteamericana, que los ciudadanos se vean plenamente compensados con las reparaciones.

El cálculo de débitos y créditos requiere una unidad de medida común para poder establecer su equivalencia (Lipton et al., 2008). Por ejemplo, se puede usar el número de individuos de una especie o recurso, como árboles de un determinado tipo, en el cálculo de débitos y créditos, lo que da lugar a los análisis recurso-recurso. O se puede analizar en términos de servicios que da el ecosistema afectado (análisis servicio-servicio). Otra posibilidad es considerar el valor social de los daños y la reparación, como reflejo de las pérdidas y ganancias de bienestar (análisis valor-valor). Para ello, se suele estimar primero el valor para la sociedad de la pérdida ambiental temporal mediante alguna técnica de valoración de bienes sin mercado, y luego se diseña un programa de mejora ambiental que sea percibido como del mismo valor que el débito, lo que implica un segundo ejercicio de valoración. En otras palabras, el valor del débito obtenido en el primer ejercicio da pie a la creación o diseño de un programa de restauración que se valora en un segundo ejercicio para ajustar exactamente a la cantidad de mejora ambiental (reparación compensatoria) que tenga un valor social equivalente al tamaño de los débitos. En última instancia, el análisis de equivalencia valor-valor consiste en determinar la cantidad de reparación compensatoria (crédito) que la sociedad requiere.

Este artículo muestra como ambos cálculos, débito y crédito, pueden estimarse por valoración contingente, un método que en su formato dicotómico consiste en la presentación mediante encuesta de un cambio en un bien al que se asocia un pago que la persona entrevistada acepta o rechaza (Mitchell y Carson, 1989). La ilustración corresponde a un incendio forestal en España, siendo ésta una primera aplicación de análisis de equivalencia por daños a sistemas forestales, dado que la legislación en países con mayor tradición, como los Estados Unidos, se centra principalmente en daños ambientales producidos por vertidos en agua (OPA) o daños producidos por liberación de sustancias químicas (CERCLA). No obstante, la ley Europea incluye la protección a especies endémicas, 
como el pino laricio, que es la aplicación que se presenta en este caso de estudio.

La siguiente sección expone una breve reseña del origen y revisión de la literatura sobre aplicaciones de análisis de equivalencia que usan el método de valoración contingente. Le sigue un apartado metodológico de las técnicas de valoración empleadas en este estudio y el detalle de la aplicación, tras lo cual se presentan y discuten los resultados. El artículo finaliza con unas conclusiones a modo de resumen.

\section{Revisión literaria}

La práctica de los análisis de equivalencia es de tradición relativamente reciente. En su aplicación actual, el concepto surge en la literatura a principios de los años noventa (King y Adler, 1991) en relación con un ecosistema de humedal dañado. Sin embargo, las primeras y más influyentes aplicaciones formales de análisis de equivalencia se publicaron a mediados de esa década y estaban dirigidas a análisis de daños ambientales en los servicios provistos por determinados hábitats (Unsworth y Bishop, 1994, Mazzotta et al., 1994). Estos estudios se basan en la premisa de que el público puede ser compensado por pérdidas de calidad ambiental que tuvieron lugar en el pasado mediante una provisión de servicios ambientales adicionales del mismo tipo o equivalente en el futuro. La National Oceanic and Atmospheric Administration (NOAA), de los Estados Unidos, publicó a partir de mediados de los noventa distintos documentos sobre los análisis de equivalencia que sirven de guía para su aplicación en aquel país (Dunford et al., 2004, National Oceanic and Atmospheric Administration, 1995). El uso de los análisis de equivalencia se extendió además al verse avalado por los tribunales en casos como el de daños a algas marinas del Santuario Marino Nacional de
Estados Unidos en los Cayos de Florida, en 1992, y también en daños a la flora y fauna marinas en el mismo Santuario de Florida, en 1993, causados por la rotura de una tubería submarina por un remolque marino.

Jones y Pease (1997) realizaron un primer intento por proveer de un modelo conceptual a los análisis de equivalencia. Sugerían que el uso de métodos de valoración puede ser útil en ciertos casos para la estimación en unidades monetarias del valor de un proyecto de restauración que fuera equivalente al valor de las pérdidas; es decir, el análisis de equivalencia valor-valor. Así, aunque la aplicación de la metodología inicialmente se concentró en daños de hábitat (o sea, estimando débitos y créditos en términos de servicios ambientales), esta pronto se extendió a la equivalencia recurso-recurso y valor-valor.

Una de las formas de estimar el valor de los débitos y créditos es con el método de valoración contingente (Mitchell y Carson, 1989). Algunas aplicaciones son: la valoración de la contaminación en costas de California por derrames de DDT y PCB (Montrose Settlements Restoration Program, 2005); la aplicación para estimar la compensación por daños producidos por un derrame con DDT y PCB en las costas de Southern California Bight (Carson et al., 1994); el caso de contaminación de la cuenca de Big Darby (Erekson et al., 2004); el estudio de valoración de recursos naturales dañados por contaminación en el río Athos/Delawere (Athos/ Delaware River Lost Use Technical Working Group, 2007) y la aplicación de programas de restauración en Arrecifes de Coral en Hawaii (Bishop et al., 2011). Nótese que este listado de previas aplicaciones de análisis de equivalencia con valoración contingente se refiere exclusivamente a ecosistemas acuáticos, lo que tiene que ver con que legislaciones diferentes a la que recientemente rige en la 
Unión Europea contemplan, principalmente, daños a hábitats o recursos de agua. Por ello, este estudio presenta una primera aplicación de análisis de equivalencia en un sistema forestal, si bien la metodología usada es en esencia la misma que la usada en los estudios que aquí se revisan.

En el estudio de daños a recursos marinos con derrame de DDT y PCB en las costas de Southern California Bight, Carson et al. (1994) estimaron la cantidad de compensación requerida por daños a recursos marinos que, sin intervención, tardarían 50 años en recuperarse. La pregunta de valoración siguió un formato dicotómico (de ofrecer un cambio ambiental a un precio que varía de una persona a otra y preguntar por su aceptación o rechazo, tal como se expone con mayor detalle en la próxima sección), que permitió calcular la cantidad de compensación requerida por las pérdidas producidas por los daños temporales.

Otro ejemplo es el caso de estudio para estimar los impactos del proceso de urbanización en el área de la cuenca de Big Darby Creek (Erekson et al., 2004). El ejercicio presentó cuatro escenarios de desarrollo urbano esperados para los próximos años de acuerdo a la reciente urbanización en la zona, escenarios que incluían la situación actual con un alto nivel de contaminación. Cada escenario quedaba definido por características de superficie, zona residencial, áreas arboladas y de reserva natural, y la contaminación esperada. Se preguntó a los encuestados su escenario preferido y posteriormente la pregunta de valoración donde debían elegir entre tres programas de reparación con un coste, o la situación actual sin coste adicional.
En otro caso de estudio realizado por Bishop et al. (2011) se estima el valor de un programa de protección y restauración en ecosistemas de arrecifes de Coral en Hawaii. En este estudio se utilizó el método de valoración contingente siguiendo un esquema similar al de modelos de elección que permitió valorar varios programas a la vez dentro de un mismo cuestionario. Este procedimiento les permitió obtener el valor social para distintos proyectos de reparación compensatoria para implantar posteriormente aquel de valor similar al de los débitos.

En resumen, los análisis valor-valor con el método de valoración contingente se aplican habitualmente en dos fases, una para la estimación de los débitos y otra posterior para los créditos. La estimación de créditos se realiza a menudo mediante la presentación de diversos escenarios para finalmente recomendar el programa de reparación compensatoria que sea de valor social equivalente a la pérdida de bienestar por los recursos temporalmente dañados. El estudio que aquí se presenta consiste también en dos fases. La primera, para calcular el débito, se realiza mediante una aplicación estándar de valoración contingente en formato dicotómico, a la manera tradicional. La segunda fase, para el crédito, aplica también el método de valoración contingente en su formato dicotómico, pero con una novedad en su diseño. Tradicionalmente, la cantidad de mejora ambiental se mantiene constante a lo largo de toda la muestra, y lo que varía entre submuestras es la cantidad a pagar que se le pide al individuo. La novedad consiste en variar entre submuestras la cantidad de programa de mejora ambiental (hectáreas de aforestación), manteniendo constante el pago, que se fija al valor obtenido en la primera fase; sin embargo, el formato que la persona entre- 
vistada observa en la pregunta de valoración sigue siendo el tradicional, donde se le describe la mejora ambiental y se le pide pagar una determinada cantidad de dinero para obtenerla. De esta forma se puede estimar directamente el número de hectáreas de aforestación que compensa en equivalencia el daño temporal, que es en definitiva la finalidad del ejercicio de equivalencia valor-valor.

La próxima sección muestra el fundamento teórico del formato dicotómico de valoración contingente, basado en la teoría de la maximización de la utilidad aleatoria, incluyendo la novedad de variar entre submuestras la variable física de cantidad en lugar de la monetaria de valor.

\section{Metodología}

El método de valoración contingente consiste habitualmente en simular una situación de mercado mediante un cuestionario que describe un cambio en un bien (oferta) y propone un pago para conseguirlo. La persona entrevistada declara si aceptaría o no el pago para obtener el bien (demanda). Se espera que la persona entrevistada dé su consentimiento a pagar si la utilidad que le reporta el bien no es inferior a la que le reporta la cantidad de dinero que le piden. En caso contrario, se espera que la respuesta al pago sea negativa. Es decir, se supone que las personas que participan en la encuesta se comportan racionalmente, maximizando su utilidad.

El investigador desconoce de antemano con exactitud la respuesta de la persona entrevistada (I), lo que se puede modelar mediante una función de utilidad $(U)$ de $i$ con una parte determinista y otra aleatoria (Hanemann y Kanninen, 1999). Se puede escribir como

$$
U_{i}=V_{i}+e_{i},
$$

donde $V$ corresponde a la parte determinista que el investigador puede observar y $e_{i}$ a la no observada. La introducción de la parte aleatoria da lugar al modelo de maximización de la utilidad aleatoria (McFadden, 1974).

Desarrollando [1] como función indirecta de utilidad, se obtiene que la persona entrevistada rechazaría pagar (y aceptaría en caso contrario) si

$$
U_{i}\left(p_{x^{\prime}} y_{i} z^{0}, e_{i}\right)>U_{i}\left(p_{x^{\prime}} y_{i}-c, z^{1}, e_{i}\right)
$$

donde $p_{x}$, es el vector de precios de los bienes de mercado $x ; y$ denota el nivel de ingreso o limitación presupuestaria de la persona $i ; y c$ es el pago requerido para obtener el bien $z^{1}-z^{0}$ objeto de la valoración, con el exponente 0 denotando la situación sin cambio en el bien y el 1 con cambio.

Dado que se trata de funciones con un componente aleatorio, su comparación se puede escribir en términos probabilísticos. Para la probabilidad de rechazar el pago c ( $\operatorname{Pr}\{$ no\}),

$\operatorname{Pr}\{n o\}=\operatorname{Pr}\left\{U_{i}\left(p_{x^{\prime}} y_{i}, z^{0}, e_{i}\right)>U_{i}\left(p_{x^{\prime}} y_{i}-c, z^{l}, e_{i}\right)\right\} \cdot[2]$

La máxima disposición a pagar (DAP) que la persona i desembolsaría para obtener la mejora en la provisión del bien viene definida por

$$
U_{i}\left(p_{x}, y_{i}, z^{0}, e_{i}\right)=U_{i}\left(p_{x}, y_{i}-D A P, z^{l}, e_{i}\right)
$$

Por tanto, DAP es una variable aleatoria que en este modelo varía en función de $\left(\mathrm{p}_{\mathrm{x}}, \mathrm{y}_{\mathrm{i}}, \mathrm{z}^{0}\right.$, $\mathrm{z}^{1}, \mathrm{e}_{\mathrm{i}}$ ), con lo que la ecuación [2] se puede expresar también en términos de

$\operatorname{Pr}\{n o\}=\operatorname{Pr}\left\{\operatorname{DAP}_{i}\left(p_{x}, y_{i}, z^{0}, z^{l}, e_{i}\right)<c\right\} ; \operatorname{Pr}\{s i ́\}=1-\operatorname{Pr}$ $\{$ no $\}$.

Es habitual suponer una distribución logística para la variable aleatoria DAP, aunque se pueden considerar otras (Hanemann, 1984). 
Variando $c$ a lo largo de la muestra entrevistada, se obtiene suficiente información para estimar el modelo logístico, del tipo

$$
\operatorname{Pr}\{s i ́\}=L=1 /(1+\exp (a+b c),[3]
$$

donde $L$ toma valor 1 cuando la persona responde que pagaría la cantidad de dinero $c$ y 0 en caso contrario; y a y b son los coeficientes a estimar.

Dado que la distribución logística es simétrica, el valor de su media y mediana coinciden. Es fácil deducir a partir de [3] que cuando $L$ vale $1 / 2$ el valor mediano de DAP para la muestra entrevistada es $c=-a / b$.

Lo habitual en los ejercicios de valoración contingente es que la variable monetaria c sea la que varía a lo largo de la muestra y poder obtener la media o mediana de la máxima disposición a pagar por el cambio entre $z^{0} y z^{1}$, que se mantiene constante. Sin embargo, también se puede optar por variar el nivel de cambio en el bien $z$ manteniendo fijo el pago $c$, con la misma base teórica que se acaba de exponer. Entonces la variable c de la ecuación [3] se sustituye por el cambio en $z$, y el valor mediano de $z$, que se obtiene de forma análoga $(-a / b)$, se interpreta como la mínima cantidad del bien que en promedio las personas de la muestra exigirían a cambio de pagar $c$ unidades monetarias.

\section{Aplicación}

El área de estudio se sitúa en Cataluña, región noreste de España. En 1994 un gran incendio forestal, originado por el mal funcionamiento de una línea de alta tensión, afectó aproximadamente a 25000 hectáreas, que corresponden a una tercera parte de los bosques de pino laricio (pinus nigra) que existían en Cataluña en aquel momento. Esta especie está incluida en la protección prioritaria de la Directiva Europea sobre Hábitat (Habitat
9530 de bosques Sub-Mediterranean montanae con especies endémicas de pinus nigra). Además, estos bosques representaban una opción de actividades recreativas para la población, como turismo rural, recolección de setas y otras. El análisis de equivalencia se realizó suponiendo que un caso análogo al de 1994 tuviera lugar cerca del momento de realizar el estudio, entre 2007 y 2008.

Para ello, y como ya se ha apuntado antes más brevemente, se desarrollaron dos ejercicios sucesivos de valoración contingente. La primera aplicación sirvió para estimar el valor de los daños temporales del incendio forestal hasta la recuperación primaria total del bosque, y la segunda aplicación para determinar la cantidad de reparación compensatoria equivalente, es decir, el tamaño del programa de restauración que tendría un valor social equivalente al de los débitos hallado en la primera aplicación. Las características específicas del bosque incendiado eran conocidas porque habían quedado registradas en el Inventario Ecológico y Forestal de Cataluña y el Segundo Inventario Forestal Nacional que se habían tomado un año antes de ocurrir el gran incendio forestal en la zona de interés, lo cual facilitó la información que se proporcionó en los dos cuestionarios.

Con el fin de estimar el valor del débito, el primer cuestionario planteó un programa de prevención de incendios para evitar un gran incendio forestal muy parecido al que sucedió en los años noventa y proponía un pago para llevarlo a la práctica. Así, el cambio a valorar suponía un programa de prevención de incendios para preservar y proteger esta zona de bosques de pino laricio considerados de alto riesgo de incendio, en lugar de referirse directamente al incendio ya acontecido en 1994. La valoración ex ante, simulando la reincidencia en el daño de interés, es la práctica habitual aceptada en estudios 
de valoración de daños a recursos naturales (Carson et al., 2003, Arrow et al., 1993).

El cuestionario se organizó en tres apartados; el primero presentó el cambio a valorar, introdujo los tipos de bosque más abundantes en Cataluña incluidos los bosques de laricio y se comprobó la familiaridad del encuestado con este tipo de bosque. A continuación se describió la situación específica sobre el bosque que se pretendía preservar, la densidad del bosque y la alta probabilidad de un incendio forestal en los próximos años así como las consecuencias. El cuestionario también enfatizó que en caso de ocurrir el incendio el bosque tardaría 50 años en recuperarse, es decir los daños temporales. La información reflejó las características que habían tenido lugar en el pasado, las dimensiones de aquel incendio, la región afectada, las especies, y el tiempo que tardaría el bosque en recuperar su estado original.

El segundo apartado orientó gradualmente al escenario de valoración contingente, que siguió el formato dicotómico habitual. Se informó que en caso de llevar a cabo el programa de prevención de incendios éste debería ser costeado por todos los ciudadanos con pagos anuales obligatorios durante diez años. La pregunta de valoración se formuló como sigue: ¿Aceptaría pagar $x$ euros anualmente durante 10 años por un programa para prevenir un incendio que afectaría el 30\% de hectáreas de pino laricio en el centro de Cataluña?, donde lax denota una cantidad de dinero que varió de una submuestra a otra. En concreto, las cantidades o pagos presentados para el total de la muestra fueron de 10, 20, 40, 50, 60, 70, $80,100,120$ y 150 euros, y a cada persona entrevistada le correspondió un solo precio. Estas cantidades se definieron de acuerdo a las cantidades obtenidas durante las primeras versiones del cuestionario, en la fase de prueba piloto.
En el tercer apartado del cuestionario se inquirieron los motivos por los cuales estaban dispuestos a pagar tal cantidad por este programa, o bien los motivos para no querer pagar. Estas respuestas fueron de utilidad para identificar las respuestas que eran de protesta. Por último, la parte final se centró en obtener información socioeconómica de los encuestados.

Una vez llevada a cabo la estimación del débito se procedió con el segundo cuestionario para estimar la cantidad de crédito equivalente. El segundo cuestionario se organizó también en tres apartados siguiendo la estructura del primer ejercicio. El escenario propuesto consistió en un programa de aforestación de pino laricio en otro sitio para compensar y reparar a la naturaleza por la pérdida temporal del bosque en un incendio, es decir, el tiempo que tarda la reforestación en el mismo lugar en lograr un bosque como el que se tendría de no haber acontecido el incendio. El programa de aforestación compensatoria se propuso en un lugar distinto al de la zona afectada, debido a que en la zona del incendio ya estaba en proceso de recuperación tras reforestarlo. Igual que antes, el ejercicio enfatizó el concepto de pérdidas o daños temporales, indicando que un bosque de esta especie tarda unos 50 años en crecer hasta constituir un bosque maduro similar al que se quemó.

La pregunta de valoración presentó la variante del formato dicotómico del método de valoración contingente donde se fijó el pago al valor obtenido en la primera fase del estudio (el débito), mientras que la cantidad de crédito, o aforestación, variaba entre submuestras. De esta manera el segundo ejercicio estimó la cantidad mínima de compensación física que sería equivalente al valor de las pérdidas temporales (solo las temporales) producidas por el incendio de acuerdo a las percepciones sociales. La pregunta se 
formuló como sigue: Para subsanar las pérdidas temporales producidas por el incendio del $30 \%$ de bosque de pino laricio en Cataluña (señalado con rojo en el mapa) se propone un programa de forestación (área verde en el mapa) que corresponde al $x \%$ de la superficie total incendiada y tendría un coste de $60 €$ por persona durante 10 años, ¿ Estaría dispuesto a pagar $60 €$ al año durante 10 años por el programa de forestación? La cantidad de mejora ambiental, $x$, propuesta en la pregunta de valoración tomó los niveles del 10, 30, 50, 70 y 100 \%, variando de una submuestra a otra.

De esta manera, un $100 \%$ indicaba una aforestación de 25000 hectáreas adicionales a las 25000 ya reforestadas en el mismo lugar del incendio, o un $10 \%$ una superficie de 2500 hectáreas. Nótese que la forma en que una persona entrevistada observa en la pregunta de valoración es esencialmente la misma en ambos casos, sea cual sea el cuestionario al que responda: una variación en la cantidad de bosque y un pago asociado a (evitar/conseguir) dicha variación.

Ambos cuestionarios siguieron los procedimientos habituales en el diseño y la aplicación de ejercicios de valoración contingente, tal como reuniones de grupo con participantes del público en general, entrevistas individuales en mayor profundidad, cuestionarios piloto para verificar la claridad de los conceptos presentados, credibilidad de la información proporcionada, comprensión en términos de las consecuencias de un gran incendio forestal y del concepto de daños temporales, aceptación del mercado simulado en el cuestionario, y obtener unos primeros valores.

El primer cuestionario se administró durante el invierno de 2007-2008 en municipios que pertenecían a la provincia de Barcelona. Las entrevistas fueron personales, cara a cara, y realizadas a residentes mayores a 18 años. Por restricciones presupuestarias, la muestra se restringió a 300 personas, que entra dentro de los tamaños habituales en ejercicios de valoración contingente. Las personas se seleccionaron aleatoriamente respetando su representatividad en los tamaños de municipio, así como de sexo y edad según datos censales oficiales. La duración típica de las entrevistas fue de unos 14 minutos y no se detectaron problemas para la comprensión del cuestionario en general o de los conceptos de daños temporales o las consecuencias de los grandes incendios forestales.

El segundo instrumento de valoración se ejecutó de manera similar durante el primer trimestre de 2008 en la provincia de Barcelona. La muestra para este ejercicio, formada por personas no coincidentes con las de la muestra anterior, fue de 295 individuos residentes en la provincia de Barcelona y mayores de 18 años que fueron seleccionados aleatoriamente aunque respetando su representatividad en la población en términos de sexo, edad y residencia según tamaño de municipio. La duración típica de las entrevistas también fue similar y tampoco se detectaron problemas significativos en la aplicación.

\section{Resultados}

El 65\% de las respuestas al primer ejercicio de valoración se clasificaron como validas y el resto como respuestas incompletas o de protesta, que no se consideraron para la estimación del valor de los débitos. El principal motivo de protesta fue que el gobierno debería pagar por el programa. La tabla 1 contiene información sobre las características de la muestra para el programa de prevención de incendios. 
Tabla 1. Algunas características de la población y las muestras

\begin{tabular}{lccr} 
& Muestra débitos & Muestra créditos & Población \\
\hline Porcentaje hombres & 45 & 47 & 47,7 \\
Nivel Educativo (años) & 13 & 13 & 12 \\
Edad (años) & 35,4 & 34 & 39
\end{tabular}

Para la estimación de la media de la DAP se ajustó un modelo logit correspondiente a la aceptación, o rechazo, de los encuestados a pagar por el programa de prevención de incendios, como se ha explicado en la sección metodológica. La tabla 2 muestra los resultados de la estimación econométrica. El coeficiente de la variable pago es significativo y de signo negativo, indicando que la pro- babilidad de aceptar el pago propuesto para el programa de prevención de incendios disminuye con incrementos en las cantidades propuestas a pagar. La media de la máxima disposición a pagar de las personas entrevistadas se calculó según la fórmula deducida en el apartado metodológico, es decir, -coeficiente de la constante/coeficiente del pago. Es importante, por que con el signo,

Tabla 2. Resultados del modelo logit para prevención de incendios

$\begin{array}{lcc}\text { Variable } & \text { Coeficiente } & \text { Estadístico t } \\ \text { Constante } & 0.4490 & 1.56 \\ \text { Pago } & -0.0072^{\star *} & -2.04 \\ \text { Máxima Verosimilitud } & -126.03 & \\ \text { Chi-cuadrado } & 4.25 & \\ \text { N } & 185\end{array}$

** Significativo al $5 \%$

los valores de la DAP calculados se hacen positivos y esto es dinero.

Así, la DAP media anual resultó en 62.25 euros anuales por individuo, en valores de 2007, durante 10 años por la implementación de un programa para preservar 25000 hectáreas de bosques de pinos laricio en la parte central de Cataluña.

En el segundo ejercicio de valoración se utilizó, en números redondos, el valor obtenido en el primero. Así, el pago se fijó en 60 euros para toda la muestra siendo la cantidad de 
bosque la que variaba entre submuestras, como se ha explicado. El porcentaje de respuestas válidas a la pregunta de valoración en el segundo cuestionario fue de $73 \%$, tras descartar las incompletas y de protesta. Los motivos más frecuentes de las respuestas de protesta se referían a que otros, como el gobierno, o los propietarios del bosque, deberían pagar por el programa de aforestación. Estas cifras, y los motivos, no están lejos de lo habitual (véase por ejemplo Mitchell y Carson, 1989). La tabla 1 muestra algunas características de la muestra encuestada para el programa de aforestación o créditos, mientras que la tabla 3 presenta los resultados de la estimación econométrica.

El coeficiente de la variable superficie es significativo al $5 \%$ y de signo positivo, mostrando que la probabilidad de aceptar el pago de 60 euros propuesto aumenta en la medida de que el porcentaje de superficie a aforestar es mayor. La media de la cantidad mínima del programa de aforestación compensatoria que la población aceptaría por el pago de los 60 euros se calcula de la forma deducida en el apartado metodológico, como -coeficiente de la constante/coeficiente de la superficie.

Tabla 3. Resultados del modelo logit para programa de aforestación

$\begin{array}{lcc}\text { Variable } & \text { Coeficiente } & \text { Estadístico t } \\ \text { Constante } & -0.3235 & -1.33 \\ \text { Superficie } & 0.0110^{\star \star} & 2.38 \\ \text { Máxima Verosimilitud } & -145.35 & \\ \text { Chi-cuadrado } & 5.87 & \\ \text { N } & 215\end{array}$

** Significativo al 5\%

La cantidad de compensación ambiental equivalente se estimó, pues, en un $29.30 \%$ de la superficie originalmente quemada, lo que equivaldría a 7325 hectáreas. En definitiva, la reparación total del daño, tras el incendio forestal, es igual a 25000 hectáreas de plantación de pinos laricios por reparación primaria más la aforestación de una superficie adicional de casi un tercio de estos en concepto de reparación compensatoria por los daños temporales.

Tal como se ha comentado en la sección de revisión literaria, la forma habitual de proceder con valoración contingente en el segundo ejercicio, es decir de cálculo de los créditos compensatorios, es la de estimar el valor de distintos programas de mejora ambiental para seleccionar el más equivalente al valor del débito obtenido en el primer ejercicio. Alternativamente, se puede extrapolar la composición de los distintos programas valorados para componer un nuevo programa aproximadamente equivalente. Otra opción consiste en obtener el valor marginal, de una unidad, de la mejora ambiental propuesta y dimensionarla (escalarla, en el argot de los análisis de equivalencia) hasta obtener una que compense en igualdad los daños temporales. 
La primera forma de proceder conlleva el peligro de no hallar un programa lo suficientemente cercano al que se requiere para la equivalencia. La última tiene el inconveniente del mantenimiento de la proporcionalidad o scoping (Arrow et al., 1993). Es decir, puede que la DAP para aforestar una hectárea de terreno sea de $x$ euros y que la DAP para 1000 hectáreas sea notablemente distinta de 1000 veces $\mathrm{x}$, porque la utilidad marginal diste de ser constante en el tramo relevante. El procedimiento de análisis de equivalencia valor-valor presentado en este artículo muestra la aplicación de una modalidad distinta del método de valoración contingente, donde varía entre submuestras la cantidad de programa en lugar del pago, con lo que se escala directamente la cantidad de créditos que se precisa, paliando el problema del scoping.

\section{Conclusiones}

Este caso de estudio ha presentado una aplicación del análisis de equivalencia a un gran incendio forestal ocurrido en España en una zona de pino laricio. Los análisis de equivalencia en la evaluación de daños ambientales consisten en calcular la cantidad de mejora ambiental que compensa la pérdida temporal de calidad en el medio ambiente hasta que este recupera su estado básico tras un incidente. Dicho cálculo de equivalencia se puede realizar con respecto al bienestar social, y estimar mediante el método de valoración contingente.

En su aplicación habitual se realizan dos ejercicios de valoración. El primero estima el valor de los débitos o utilidad social perdida temporalmente con el incidente. El segundo estima el valor social de distintos programas de mejora ambiental esperando hallar uno que compense en equivalencia la pérdida temporal, o el valor social de un crédito o unidad de mejora para luego dimensionar la cantidad de créditos compensatorios (reparación compensatoria). Una alternativa que evita estos problemas consiste en variar la cantidad de mejora ambiental entre submuestras en el segundo ejercicio en lugar de variar el pago. En su forma, la pregunta de valoración tiene el formato dicotómico (respuesta positiva o negativa) habitual, es decir, se ofrece una mejora ambiental por la que se requiere un pago, pero la variación de la cantidad en lugar del precio permite ajustar directamente la equivalencia en términos físicos dentro del marco de equivalencia valor-valor. Una ventaja de esta opción es que evita el problema del scoping, dado que ajusta directamente la cantidad equivalente. El procedimiento es congruente con la teoría económica, como se ha mostrado en el apartado metodológico, mientras que el caso de estudio de daños forestales en España ilustra que su aplicación es viable en la práctica.

La aplicación estima el débito en unos 60 euros anuales durante 10 años (en valores de 2007, mientras que el equivalente en crédito corresponde a un programa de plantación de pino laricio de aproximadamente un 30\% de hectáreas con respecto a la superficie incendiada. Es decir, por cada hectárea incendiada el operador responsable del daño debe reponer esa misma hectárea en concepto de reparación primaria y casi un tercio de hectárea adicional en reparación compensatoria por la pérdida de bienestar temporal que se produce hasta que el nuevo bosque alcance la madurez del originalmente quemado.

\section{Agradecimientos}

La aplicación del análisis de equivalencia se realizó en el contexto del proyecto de investigación REMEDE del programa marco europeo (http://www.envliability.eu). 


\section{REFERENCIAS BIBLIOGRÁFICAS}

Arrow, K., R. Solow, P. Portney, E. Leamer, R. Radner \& H. Schuman (1993) "Report of the NOAA panel on contingent valuation", en Federal Register, 58, 4602-4614.

Athos/Delaware river lost use technical working group (2007) "Damage Assessment, Remediation and Restoration Program. National Oceanic and Atmospheric Admnistration", en Athos/Delaware River Lost Use Valuation Report. Athos/Delaware River Trustee Council.

Bishop, R. C., D. Chapman, BJ. Kanninen, JA Krosnick, B. Leeworthy, \& NF. Meade (2011) "Total Economic Value for Protecting and Restoring Hawaiian Coral Reef Ecosystems. Final Report", en NOAA Technical Memorandum CRCP. Silver Spring, MD: NOAA Office of National Marine Sanctuaries, Office of Responses and Restoration, and Coral Reef Conservation Program.

Carson, R T., MW. Hanemann, R. Kopp, JA. Krosnick, RC. Mitchell, S. Presser, PA. Ruud \& KV. Smith (1994) "Prospective Interim Lost Use Value due to DDT and PCB Contamination in the Southern California Bight", en: ADMINISTRATION, N. O. A. A. (ed.).

Carson, RT., RC. Mitchell, MW. Hanemann, R. Kopp, S. Presser \& PA. Ruud (2003) "Contingent Valuation and loss passive use: Damages from the Exxon Valdez oil spill", en Environmental and Resource Economics, 25, 257-286.

CERCLA (1980) "Comprehensive Environmental Response, Compensation and Liability Act. U.S." En Code Title 42, Chapter 103.
Council of the European Parliament (2004) "Environmental Liability Directive. 2004/35/ EC".

Dunford, RW., TC. Ginn \& WH. Desvousges (2004) "The use of habitat equivalency analysis in natural resource damage assessments", en Ecological Economics, $48,49-70$.

Erekson, OH., OL. Loucks, SR. Elliot, DS. Mccollum, M. Smith \& RJF. Brunis (2004) "Evaluating Development Alternatives for a High-Quaility Stream threatened by urbanization: Big Darby Creek Watershed", en Brunis, Randakk JF. \& Heberling, Matthew T. (eds.) Economics and Ecological Risk Assessment. Boca Raton: CRC Press.

Hanemann, MW (1984) "Discrete/Continuous Models of Consumer Demand", en Econometrica, 52, 541-562.

Hanemann, MW \& BJ. Kanninen (1999) "The statistical analysis of discrete-response CV data", en Bateman, IJ \& KG. willis, (eds.) Valuing Environmental Preferences. Theory and Practice of the Contingent Valuation Method in the US, EU, and Developing Countries. Oxford: Oxford University Press.

Jones, CA \& KA. Pease (1997) "Restorationbased compensation measures in natural resource liability statutes", en Contemporary Economic Policy 15, 111-122.

King, DM \& KJ. Adler (1991) Scientifically defensible compensation ratios for wetlands mitigation, Washington, DC, U.S. Environmental Protection Agency Office of Public, Planning and Evaluation.

Lipton, J., K. Lejeune, J-B. Calewaert \& E. 
Ozdemiroglu (2008) Toolkit for Performing Resource Equivalency Analysis to Assess and Scale Environmental Damage in the European Union. London: EFTEC.

Mazzotta, MJ., JJ. Opaluch \& TA. Grigalunas (1994) "Natural resource damage assessment: The role of resource restoration", en Natural Resources Journal, 34, 153-178.

Mcfadden, D. (1974) "Conditional logit analysis of qualitative choice behavior", en: Zarembka, P. (ed.) Frontiers in Econometrics. New York: Academic Press.

Mitchell, RC \& R. Carson (1989) Using surveys to value public goods: the contingent valuation method, Washington, D.C. [Baltimore], Resources for the Future; Johns Hopkins University Press [distributor].

Montrose settlements restoration program (2005) "Final restoration plan and programmatic environmental impact statement, and environmental impact report" En Unpublished report. National Oceanic and Atmos- pheric Administration, Department of Parks and Recreation, and California State Lands Commission.

National oceanic and atmospheric administration (1995) Habitat Equivalency Analysis: An Overview. Washington, D.C.: Damage Assessment and Restoration Program, NOAA.

Opa (1990). Oil Pollution Act. U.S. Code Title 33, Chapter 40.

Unsworth, RE \& RC. Bishop (1994) "Assessing natural resource damages using environmental annuities", en Ecological Economics, 11, 35-41. 